\title{
Bandlimited Signal Reconstruction From the Distribution of Unknown Sampling Locations
}

\author{
Animesh Kumar \\ Department of Electrical Engineering \\ Indian Institute of Technology Bombay \\ Mumbai, India - 400076 \\ Email: animesh@ee.iitb.ac.in
}

\begin{abstract}
We study the reconstruction of bandlimited fields from samples taken at unknown but statistically distributed sampling locations. The setup is motivated by distributed sampling where precise knowledge of sensor locations can be difficult.

Periodic one-dimensional bandlimited fields are considered for sampling. Perfect samples of the field at independent and identically distributed locations are obtained. The statistical realization of sampling locations is not known. First, it is shown that a bandlimited field cannot be uniquely determined with samples taken at statistically distributed but unknown locations, even if the number of samples is infinite. Next, it is assumed that the order of sample locations is known. In this case, using insights from order-statistics, an estimate for the field with useful asymptotic properties is designed. Distortion (meansquared error) and central-limit are established for this estimate.
\end{abstract}

\section{INTRODUCTION}

In the smart-dust paradigm [1], consider a distributed field sampling problem where sensors are deployed without precise control on the sensor-locations. One method for distributed field sampling is to learn the location of these individual sensors, and then reduce field acquisition to the well-studied non-uniform sampling problem [2]. However, localization of individual sensors in a wireless sensor network can be difficult [3]. In light of these issues, the reconstruction of a physical field from samples taken at unknown but statistically distributed locations is studied in this work.

Assuming that the field has a finite support, sensors will have to be deployed in the finite region where the field is nonzero. The smoothness of the physical field can be modeled by bandlimitedness. In this work, it will be assumed that the field is spatially periodic and bandlimited. Only onedimensional fields will be considered. The lack of control in sensor deployment is modeled by a uniform-distribution on the sensor or sampling-locations. It is assumed that sensors are deployed (or scattered) independent of each other. Thus, perfect samples of the field at independent and identically distributed (i.i.d.) but also unknown locations are obtained by the sampling method outlined above. From these samples the field has to be estimated. This work focuses on a consistent estimate, that is, an estimate which converges to the true underlying field when the number of samples is infinite.

This work has been supported by grant no. P09IRCC039, IRCC, IIT Bombay.
The key results shown in this work are as follows:

1) It will be shown that a bandlimited field cannot be uniquely determined with perfect samples obtained at statistically distributed locations, even if the number of samples is infinite.

2) If the order of sample locations is known, then using insights from classical order-statistics, a consistent estimate for the spatial field is presented. Distortion (average mean-squared error) and a central-limit type weak convergence result are established for this estimate.

Prior art: Recovery of discrete-time bandlimited signals from samples taken at unknown locations was first studied by Marziliano and Vetterli [4]. Recovery of a bandlimited signal from a finite number of ordered nonuniform samples at unknown sampling locations has been studied by Browning [5]. Estimation of periodic bandlimited signals in the presence of random sampling location under two models has been studied by Nordio et al. [6]. Their first model studies reconstruction of bandlimited signal affected by noise at random but known locations. Their second model studies estimation of bandlimited signal from noisy samples on a location set obtained by random perturbation of equi-spaced deterministic grid.

In contrast, this work presents the estimation of a bandlimited field from i.i.d. distributed but unknown samples in an asymptotic setting (where the number of samples increases to infinity). Asymptotic consistency (convergence in probability), mean-squared error bounds, and central-limit type weak law are the focus of this work. The first key-result of this work is absent in related work due to difference in the sensing model. Organization: In Section $\amalg$ the field model, distortion, sensor deployment model, and useful statistical theory are outlined. In Section IIII asymptotic consistency, mean-squared error, and weak convergence aspects of field estimate are discussed. Finally, conclusions will be presented in Section IV

\section{PRoblem SETUP AND USEFUl ClASSiCAL RESUltS}

This section will review the field model, the distortion, and some useful mathematical results. Field model appears first.

\section{A. Field model and associated properties}

The field of interest $g(t)$ is periodic, real-valued, and bandlimited. Without loss of generality, the period is assumed to be $T=1$. It is also assumed that the field $|g(t)| \leq 1$ is 
bounded. Bandlimitedness implies that some $b>0$ coefficients are non-zero in the Fourier series. Thus,

$$
g(t)=\sum_{k=-b}^{b} a_{k} \exp (j 2 \pi k t) .
$$

Real-valued $g(t)$ implies conjugate symmetry in the Fourier domain, that is, $a_{k}=a_{-k}^{*}$; however, this symmetry will not be utilized in this work. The $(b+1)$ Fourier coefficients constitute the degrees of freedom for this signal. With $\|g\|_{\infty} \leq 1$, using Bernstein's inequality [7], we get

$$
\left|g^{\prime}(t)\right| \leq 2 \pi b
$$

where $2 \pi b \mathrm{rad} / \mathrm{s}$ is the bandwidth of the signal. For simplicity of notation, define $s_{b}:=1 /(2 b+1)$ as a spacing parameter and $\phi_{k}:=\exp (j 2 \pi k /(2 b+1))=\exp \left(j 2 \pi k s_{b}\right)$. By using $(2 b+1)$ samples of the field $g(t)$, its Fourier coefficients can be computed as follows:

$$
\left[\begin{array}{c}
g(0) \\
g\left(s_{b}\right) \\
\vdots \\
g\left(2 b s_{b}\right)
\end{array}\right]=\left[\begin{array}{ccc}
1 & \cdots & 1 \\
\phi_{-b} & \cdots & \phi_{b} \\
\vdots & & \vdots \\
\left(\phi_{-b}\right)^{2 b} & \cdots & \left(\phi_{b}\right)^{2 b}
\end{array}\right]\left[\begin{array}{c}
a_{-b} \\
a_{-b+1} \\
\vdots \\
a_{b}
\end{array}\right]
$$

or more simply

$$
\vec{g}=\Phi_{b} \vec{a},
$$

where the vector matrix notation is obvious. The columns of $\Phi_{b}$ are orthogonal with a norm-square $(2 b+1)$ under the standard inner-product. The relation in (3) is inverted to obtain

$$
\vec{a}=\left(\Phi_{b}\right)^{-1} \vec{g}=\frac{1}{(2 b+1)} \Phi_{b}^{\dagger} \vec{g}
$$

where $\Phi_{b}^{\dagger}$ is the conjugate transpose of $\Phi_{b}$. The expression in (4) will be used to obtain an estimate for $\vec{a}$ as discussed later.

\section{B. Sensor deployment model and reconstruction distortion}

Denote any sequence as $x_{l}^{m}:=\left(x_{l}, x_{l+1}, \ldots, x_{m}\right)$ for $m \geq l$. It will be assumed that sensors are deployed at random locations $U_{1}^{n}$ in the interval of interest $[0,1]$. The locations $U_{1}^{n}$ are i.i.d. random variables with uniform distribution and probability density function $f(u)=1$ for $0 \leq u \leq 1$. The locations $U_{1}^{n}$ are not known in our model. An asymptotic number of samples and limiting distribution of $U_{1}^{n}$ will be used for field estimation. The average mean-squared error will be used as a distortion metric. If $\widehat{G}(t)$ is any estimate of $g(t)$, then the distortion is defined as

$$
D:=\mathbb{E}\left(\|\widehat{G}-g\|_{2}^{2}\right):=\mathbb{E}\left[\int_{0}^{1}|\widehat{G}(t)-g(t)|^{2} \mathrm{~d} t\right] .
$$

\section{Useful mathematical results}

For estimation of field from the statistical properties of $U_{1}^{n}$, the following convergence results will be useful. These results for order-statistics and quantiles are a counterpart to the strong-law of large numbers (see [8, Ch. 10]). The ordered version of $U_{1}^{n}$ will be denoted by $U_{1: n}^{n: n}:=\left\{U_{1: n}, U_{2: n}, \ldots, U_{n: n}\right\}$ where $U_{n: n}$ is the largest and $U_{1: n}$ is the smallest [8].
For uniform distribution, the $p$-th population quantile $q_{p}$ is equal to $p$. Then with $r=[n p]+1$, it is known that [8. pg. 285]

$$
U_{r: n}-p=-\left(F_{n}(p)-p\right)+R_{n}
$$

where $F_{n}(u):=\frac{1}{n} \sum_{i=1}^{n} \mathbb{1}\left(U_{i} \leq u\right)$ is the empirical distribution of $U_{1}^{n}$. The remainder term $R_{n}$ decreases to 0 almost surely,

$$
R_{n}=O\left(n^{-3 / 4}(\log n)^{1 / 2}(\log \log n)^{1 / 4}\right) \quad \text { as } n \rightarrow \infty .
$$

By the strong law of large numbers [9], we know that $F_{n}(p) \stackrel{a . s}{\longrightarrow} p$; thus, $U_{r: n} \stackrel{a . s .}{\longrightarrow} p$ from (7). Analogous to the central limit theorem, the following fact is noted.

Fact 2.1: [8, Theorem 10.3] Let $0<p_{1}<p_{2}<\ldots<$ $p_{2 b+1}<1$ and assume that $\left(r_{i} / n-p_{i}\right)=o(1 / \sqrt{n}), i=$ $1,2, \ldots, 2 b+1$. Then the following result holds:

$$
\sqrt{n}\left[U_{r_{1}: n}-p_{1}, \ldots, U_{r_{2 b+1}: n}-p_{2 b+1}\right]^{T} \stackrel{d}{\longrightarrow} \mathcal{N}\left(\overrightarrow{0}, K_{U}\right),
$$

where $\left[K_{U}\right]_{j, j^{\prime}}=p_{j}\left(1-p_{j^{\prime}}\right)$ for $j \leq j^{\prime}$.

All the moments of $U$ are finite since it is bounded (by definition). The second moment of $U_{r: n}-p$, with $r \approx[n p]$ is bounded by.

$$
\begin{aligned}
n \mathbb{E}\left(U_{r: n}-p\right)^{2} & =p(1-p) \mathbb{E}\left(Z^{2}\right)+O(\sqrt{1 / n}), \\
& \leq \frac{1}{4}+O(\sqrt{1 / n}) .
\end{aligned}
$$

where $Z \sim \mathcal{N}(0,1)$ is a normalized Gaussian random variable.

The following fact relates consistency and $\mathcal{L}^{2}$ convergence.

Fact 2.2: [9] If $X_{n} \stackrel{\text { a.s. }}{\longrightarrow} X$ and $Y_{n} \stackrel{\text { a.s. }}{\longrightarrow} Y$, then $a X_{n}+$ $b Y_{n} \stackrel{a . s}{\longrightarrow} a X+b Y$ for any constants $a, b \in \mathbb{R}$. If $X_{n}$ is bounded and $X_{n} \stackrel{a . s}{\longrightarrow} X$, then $X_{n} \stackrel{\mathcal{L}^{2}}{\longrightarrow} X$.

We now proceed to the main results of this paper.

\section{SAMPLING AND ESTIMATION WITH RANDOM SAMPLES}

In this section, the key results of this work are presented. It will be shown that the field $g(t)$ cannot be inferred uniquely from samples collected at $U_{1}^{\infty}$, where sample-locations are unknown. Further, with order information on sample-locations, consistent estimation of the field is presented.

\section{A. It is impossible to infer $g(t)$ uniquely from $U_{1}^{\infty}$}

It will be shown that if $g\left(U_{1}\right), \ldots, g\left(U_{n}\right)$ is available without the knowledge of $U_{1}^{n}$, then $g(t)$ cannot be inferred uniquely as $n \rightarrow \infty$. Consider the statistic

$$
F_{g, n}(x)=\frac{1}{n} \sum_{i=1}^{n} \mathbb{1}\left(g\left(U_{i}\right) \leq x\right),
$$

where $\mathbb{1}()$ are the indicator random variables. Then $F_{g, n}(x), x \in[-1,1]$ completely characterizes the field values $g\left(U_{1}\right), \ldots, g\left(U_{n}\right)$ and vice-versa. By Glivenko-Cantelli theorem, the right hand limit in 9 converges almost surely to $\mathbb{P}(g(U) \leq x)$ for all $x \in[-1,1]$ as $n \uparrow \infty$ [10]. This limit is explained using Fig. 1. For any $x \in[-1,1]$ the set of points where $g(t) \leq x$ can be marked on the $t$-axis. The length or measure of this set is equal to $\mathbb{P}(g(U) \leq x)$. 


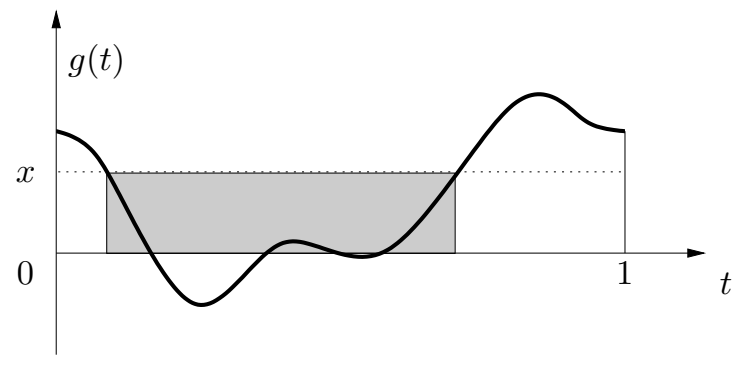

Fig. 1. For any $x \in[-1,1]$ the set of points where $g(t) \leq x$ can be marked on the $t$-axis. The length or measure of this set is equal to $\mathbb{P}(g(U) \leq x)$.

For $0<\theta<1$, let $g_{\theta}(t)=g(t-\theta)$, i.e., $g_{\theta}(t)$ is the shifted version of $g(t)$. Since $g(t)$ is periodic, its shifts will be cyclic in nature in the period $[0,1]$. Thus, the level-sets of $g(t-\theta)$ will be cyclic (in $\theta)$ and its measure $\left\{u: g_{\theta}(u) \leq x\right\}$ will be independent of $\theta$ for every $x \in[-1,1]$. Therefore, $\mathbb{P}\left(g_{\theta}(U) \leq x\right)$ will be independent of $\theta$ for every $x \in[-1,1]$. Thus, by only using $F_{g, n}(x)$, which converges to $\mathbb{P}(g(U) \leq$ $x), x \in[-1,1]$, the field $g(t)$ cannot be inferred uniquely. This completes the discussion of this subsection.

\section{B. Consistent estimation of $g(t)$ from $U_{1: n}^{n: n}$}

From now on, it will be assumed that order information of samples is available. That is, samples $g\left(U_{1: n}\right), \ldots, g\left(U_{n: n}\right)$ are available and $g(t)$ has to be estimated. Using (4) and the convergence results in Sec. II-C the following estimate for the Fourier series coefficients of $g(t)$ is proposed:

$$
\vec{A}:=\left[\widehat{A}_{-b}, \widehat{A}_{-b+1}, \ldots, \widehat{A}_{b}\right]^{T}:=\frac{1}{(2 b+1)} \Phi_{b}^{\dagger} \vec{G} .
$$

where $\vec{G}=\left[g\left(U_{1: n}\right), g\left(U_{\left[n s_{b}\right]+1: n}\right), \ldots, g\left(U_{\left[n 2 b s_{b}\right]+1: n}\right)\right]^{T}$. With (6) and the smoothness properties (continuity) of $g(t)$, this estimate is obtained by substitution method in (4). Using $\vec{A}$, an estimate for $g(t)$ is obtained as follows

$$
\widehat{G}(t)=\sum_{k=-b}^{b} \widehat{A}_{k} \exp (j 2 \pi k t)=\Phi(t)^{T} \vec{A}
$$

where $\Phi(t)^{T}=\left[\begin{array}{lll}\exp (-j 2 \pi b t) & \ldots & \exp (j 2 \pi b t)\end{array}\right]$. Intuitively, $g(t)$ has a finite degrees of freedom. This enables a procedure to estimate the Fourier series coefficients (the degrees of freedom) from a finite number of sample estimates of $g(t)$. Using these estimates of the Fourier series coefficients, the entire field of interest $g(t)$ can be estimated. For distortion calculation, the Parseval's theorem [11] will be useful,

$$
\|\widehat{G}-g\|_{2}^{2}=\sum_{k=-b}^{b}\left|\widehat{A}_{k}-a_{k}\right|^{2} .
$$

A bound on $\mathbb{E}\left(\left|\widehat{A}_{k}-a_{k}\right|^{2}\right)$ will result in a bound on the expected mean-squared error $\mathbb{E}\left(\|\widehat{G}-g\|_{2}^{2}\right)$.

We state our first result now.

Theorem 3.1 (Consistency of $\vec{A}$ ): Let $U_{1: n}^{n: n}$ be ordered i.i.d. Uniform $[0,1]$ random variables. Define $\vec{A}$ and $\widehat{G}(t)$ as in
(10) and 111. Then the estimates $\vec{A}$ and $\widehat{G}(t)$ are consistent in almost-sure and $\mathcal{L}^{2}$ sense to their respective limits, i.e.,

$$
\vec{A} \stackrel{a . s .}{\longrightarrow} \vec{a}, \widehat{G}(t) \stackrel{a . s}{\longrightarrow} g(t) \text { and } \vec{A} \stackrel{\mathcal{L}^{2}}{\longrightarrow} \vec{a}, \widehat{G}(t) \stackrel{\mathcal{L}^{2}}{\longrightarrow} g(t) .
$$

Proof: Only a sketch is provided due to space constraints. First note that $U_{\left[n i s_{b}\right]+1: n} \stackrel{a . s}{\longrightarrow} i s_{b}$ for each $i=0,1, \ldots, 2 b$. Since $g(t)$ is continuous by assumption, $g\left(U_{\left[n i s_{b}\right]+1: n}\right) \stackrel{a . s}{\longrightarrow} g\left(i s_{b}\right)$ for each $i=0,1, \ldots, 2 b$. Let $\vec{G}:=\left[g\left(U_{1: n}\right), g\left(U_{\left[n s_{b}\right]+1: n}\right), \ldots, g\left(U_{\left[n 2 b s_{b}\right]+1: n}\right)\right]^{T}$ and $\vec{g}:=$ $\left[g(0), g\left(s_{b}\right), \ldots, g\left(2 b s_{b}\right)\right]^{T}$. By repeated use of Fact 2.2 any finite linear combination $\vec{c}^{T} \vec{G}$ converges almost-surely to $\vec{c}^{T} \vec{g}$. Thus, from 10, each element of $\vec{A}$ converges almost surely to $\vec{a}$. Hence, $\vec{A} \stackrel{a . s}{\longrightarrow} \vec{a}$.

Next, $\widehat{G}(t)$ is a finite linear combination of $\vec{A}$. Since $\vec{A} \stackrel{a . s}{\longrightarrow}$ $\vec{a}$, therefore, $\widehat{G}(t) \stackrel{a . s}{\longrightarrow} g(t)$ in a similar fashion as above.

For $\mathcal{L}^{2}$-convergence, note that $\vec{G}$ is bounded in each coordinate since $|g(t)| \leq 1$ for all $t$. Each element of the matrix $\Phi_{b}$ has a magnitude one. Thus, by (10) and the triangle inequality, $\left|\widehat{A}_{i}\right| \leq\|g\|_{\infty} \leq 1$ for every $i=-b,-b+1, \ldots, b$. Thus, each $\widehat{A}_{i}$ is a bounded random variable. For bounded random sequences, from Fact 2.2, $\vec{A} \stackrel{a . s}{\longrightarrow} \vec{a}$ implies that $\vec{A} \stackrel{\mathcal{L}^{2}}{\longrightarrow} \vec{a}$. Similarly, $|\widehat{G}(t)| \leq \sum_{k=-b}^{b}\left|\widehat{A}_{k}\right| \leq(2 b+1)$ from 111. Thus, by Fact2.2 $\widehat{G}(t) \stackrel{\text { a.s. }}{\longrightarrow} g(t)$ implies $\widehat{G}(t) \stackrel{\mathcal{L}^{2}}{\longrightarrow} g(t)$, since $\widehat{G}(t)$ is bounded.

The second result establishes the scaling of distortion for the proposed estimate in (11).

Theorem 3.2: Let $U_{1: n}^{n: n}$ be ordered i.i.d. Uniform $[0,1]$ random variables. Define $\overrightarrow{\vec{A}}$ and $\widehat{G}(t)$ as in (10) and (11). Then,

$$
n \mathbb{E}\left[\|\widehat{G}-g\|_{2}^{2}\right] \leq \pi^{2} b^{2}(2 b+1)[1+O(\sqrt{1 / n})],
$$

that is, the expected distortion decreases as $O(1 / n)$.

Proof: The proof is presented in two parts. First, using the smoothness properties of $g(t)$, the norm $\|\widehat{G}-g\|_{2}^{2}$ will be bounded using the error in quantiles $U_{[n p]+1: n}-p$. Next, the convergence rate of $U_{[n p]+1: n}-p$ as in (8) will be utilized to upper-bound the distortion. First note that

$$
\begin{aligned}
\|\widehat{G}-g\|_{2}^{2} & =\sum_{k=-b}^{b}\left|\widehat{A}_{k}-a_{k}\right|^{2} \\
& =\frac{1}{(2 b+1)^{2}}\left\|\Phi_{b}^{\dagger}(\vec{G}-\vec{g})\right\|_{2}^{2} \\
& =\frac{1}{(2 b+1)^{2}} \sum_{k=-b}^{b}\left|\sum_{l=0}^{2 b}\left[\phi_{k}^{l}\right]^{*}\left(\widehat{G}\left(l s_{b}\right)-g\left(l s_{b}\right)\right)\right|^{2} \\
& \left.\stackrel{(a)}{\leq} \frac{(2 b+1)}{(2 b+1)^{2}} \sum_{k=-b}^{b} \sum_{l=0}^{2 b} \mid \phi_{k}^{l} \| \widehat{G}\left(l s_{b}\right)-g\left(l s_{b}\right)\right)\left.\right|^{2} \\
& \left.\stackrel{(b)}{=} \frac{1}{(2 b+1)} \sum_{k=-b}^{b} \sum_{l=0}^{2 b} \mid \widehat{G}\left(l s_{b}\right)-g\left(l s_{b}\right)\right)\left.\right|^{2} \\
& \left.\stackrel{(c)}{\leq} \sum_{l=0}^{2 b} \mid \widehat{G}\left(l s_{b}\right)-g\left(l s_{b}\right)\right)\left.\right|^{2} .
\end{aligned}
$$




$$
\begin{aligned}
& \left.=\sum_{l=0}^{2 b} \mid g\left(U_{\left[n l s_{b}\right]+1: n}\right)-g\left(l s_{b}\right)\right)\left.\right|^{2} . \\
& \leq\left\|g^{\prime}\right\|_{\infty}^{2} \sum_{l=0}^{2 b}\left|U_{\left[n l s_{b}\right]+1: n}-l s_{b}\right|^{2} .
\end{aligned}
$$

where $(a)$ follows by $\left(a_{1}+a_{2}+\ldots+a_{n}\right)^{2} \leq n\left(a_{1}^{2}+a_{2}^{2}+\right.$ $\left.\ldots+a_{n}^{2}\right),(b)$ follows by $\left|\phi_{k}\right|=1$ for all $k$, and $(c)$ follows since the summation does not depend on $k$. Using (8), and taking expectations on both sides

$$
\begin{aligned}
n \mathbb{E}\left(\|\widehat{G}-g\|_{2}^{2}\right) & \leq\left\|g^{\prime}\right\|_{\infty}^{2} \sum_{l=0}^{2 b} n \mathbb{E}\left(\left|U_{\left[n l s_{b}\right]+1: n}-l s_{b}\right|^{2}\right) \\
& \leq\left\|g^{\prime}\right\|_{\infty}^{2} \sum_{l=0}^{2 b}\left[\frac{1}{4}+O(\sqrt{1 / n})\right] \\
& \leq(2 \pi b)^{2}(2 b+1) \frac{1}{4}+O(\sqrt{1 / n}) \\
& =\pi^{2} b^{2}(2 b+1)[1+O(\sqrt{1 / n})]
\end{aligned}
$$

This completes the proof.

The third result establishes the weak-convergence of $\widehat{G}(t)$.

Theorem 3.3 (Central limit for $\widehat{G}(t)$ ): Let $U_{1: n}^{n: n}$ be ordered i.i.d. Uniform $[0,1]$ random variables and $\vec{u}=$ $\left(0, s_{b}, 2 s_{b}, \ldots 2 b s_{b}\right)^{T}$. Define $\vec{A}$ and $\widehat{G}(t)$ as in (10) and (11). Then the estimate $\vec{A}$ and $\widehat{G}(t)$ satisfy the following central limits:

$$
\sqrt{n}(\vec{A}-\vec{a}) \stackrel{d}{\longrightarrow} \mathcal{N}\left(\overrightarrow{0}, K_{A}\right)
$$

where $K_{G}=\nabla g^{T}(\vec{u}) K_{U} \nabla g(\vec{u})$ and $K_{\vec{A}}$ is independent of $n$ and given in terms of $K_{G}$ and $\Phi_{b}$. Further,

$$
\sqrt{n}(\widehat{G}(t)-g(t)) \stackrel{d}{\longrightarrow} \mathcal{N}\left(\overrightarrow{0}, K_{G}(t)\right) .
$$

where $K_{G}(t)$ is independent of $n$ and given in terms of $K_{G}$ and $\Phi_{b}$.

Proof: From Fact 2.1, we know that $\vec{U}:=$ $\left[U_{1: n}, U_{\left[n s_{b}\right]+1: n}, \ldots, U_{\left[n 2 b s_{b}\right]+1: n}\right]^{T} \quad$ is asymptotically normal. That is, $\sqrt{n}(\vec{U}-\vec{u}) \stackrel{d}{\longrightarrow} \mathcal{N}(\overrightarrow{0}, K)$, where

$$
[K]_{i, i^{\prime}}=(i-1) s_{b}\left[1-\left(i^{\prime}-1\right) s_{b}\right] \text { for } i \leq i^{\prime} .
$$

Note that $[K]_{i, i^{\prime}}=[K]_{i^{\prime}, i}$ by the symmetry of a covariance matrix. Recall $\vec{G}$ from (10). Since $g(t)$ is a differentiable field, by the delta-method [10],

$$
\sqrt{n}(\vec{G}-\vec{g}) \stackrel{d}{\longrightarrow} \mathcal{N}\left(\overrightarrow{0}, K_{\vec{G}}\right),
$$

where $K_{\vec{G}}=\nabla g(\vec{u})^{T} K \nabla g(\vec{u})$. Observe that the matrix $K_{\vec{G}}$ depends on the field $g(t)$. However, by smoothness of $g(t)$, the vector $\nabla g(\vec{u})$ is bounded and $K$ is independent of $n$. Thus, $K_{\vec{G}}$ is independent of $n$. From (10), since $\vec{A}$ is obtained from $\vec{G}$ by a complex-valued linear transformation, we get

$$
\sqrt{n}(\vec{A}-\vec{a}) \stackrel{d}{\longrightarrow} \mathcal{C N}\left(\overrightarrow{0}, K_{\vec{A}}\right) .
$$

Observe that the limit is a complex normal Gaussian vector. In general, the covariance properties of a zero-mean complex random vector $\vec{S}$ are determined by $\mathbb{E}\left(\vec{S} \vec{S}^{\dagger}\right)$ and $\mathbb{E}\left(\vec{S} \vec{S}^{T}\right)$. Thus,
$K_{\vec{A}}$ is determined by the two matrices $\frac{1}{(2 b+1)^{2}} \Phi_{b}^{\dagger} K_{\vec{G}} \Phi_{b}$ and $\frac{1}{(2 b+1)^{2}} \Phi_{b}^{\dagger} K_{\vec{G}} \Phi_{b}^{T}$. The covariance matrix $K_{\vec{G}}$ is independent of $n$; therefore, $K_{\vec{A}}$ is also independent of $n$ and well defined.

Finally, $\widehat{G}(t)$ is obtained from $\vec{A}$ by a $t$-dependent inner product. From (11), we get $\widehat{G}(t)=\Phi(t)^{T} \vec{A}$. Therefore, $\widehat{G}(t)$ is a complex normal Gaussian vector. Its variance can be determined by $\mathbb{E}\left(\widehat{G}(t)^{2}\right)$ and $\mathbb{E}\left(|\widehat{G}(t)|^{2}\right)$ which are equal to $\frac{1}{(2 b+1)^{2}} \Phi(t)^{T} \Phi_{b}^{\dagger} K_{\vec{G}} \Phi_{b}^{T} \Phi(t)$ and $\frac{1}{(2 b+1)^{2}} \Phi(t)^{T} \Phi_{b}^{\dagger} K_{\vec{G}} \Phi_{b} \Phi(t)^{\dagger}$, respectively. Thus the proof is complete.

This completes our technical result section. The estimation technique outlined in this section holds well for noise-free setting. If there is additive noise affecting the samples, then more involved estimation techniques will be required. Obtaining consistent estimates for $g\left(l s_{b}\right), l=0, \ldots,(2 b+1)$ is more challenging in the presence of noise.

\section{CONCLUSIONS}

The reconstruction of bandlimited fields from samples taken at unknown but statistically distributed sampling locations was studied. Periodic one-dimensional bandlimited fields were considered for sampling. Perfect samples of the field at i.i.d. uniform locations were used for the reconstruction. It was shown that a bandlimited field cannot be uniquely determined only with samples taken at statistically distributed locations, even if the number of samples is infinite. Using order information on the sample locations, a consistent estimate was proposed for the underlying field. It was shown that this estimate converges in the mean-squared error sense and almost-sure sense. Further, the mean-squared error asymptotically decreases as $O(1 / n)$, where $n$ is the number of obtained field samples.

\section{REFERENCES}

[1] J. M. Kahn, R. H. Katz, and K. S. J. Pister, "Next century challenges: Mobile networking for "smart dust"," in ACM International Conference on Mobile Computing and Networking (MOBICOM), Aug 1999, pp. 271-278. [Online]. Available: citeseer.nj.nec.com/kahn99next.html

[2] Farokh Marvasti (ed.), Nonuniform Sampling. New York, USA: Kluwer Academic Publishers, 2001.

[3] N. Patwari, J. N. Ash, S. Kyperountas, A. O. H. III, R. L. Moses, and N. S. Correal, "Location the nodes: Cooperative localization in wireless sensor networks," IEEE Signal Processing Magazine, vol. 22, no. 4, pp. 54-69, Jul. 2005.

[4] P. Marziliano and M. Vetterli, "Reconstruction of irregularly sampled discrete-time bandlimited signals with unknown sampling locations," IEEE Transactions on Signal Processing, vol. 48, no. 12, pp. 3462 3471, Dec. 2000.

[5] J. Browning, "Approximating signals from nonuniform continuous time samples at unknown locations," IEEE Transactions in Signal Processing, vol. 55, no. 4, pp. 1549-1554, Apr. 2007.

[6] A. Nordio, C.-F. Chiasserini, and E. Viterbo, "Performance of linear field reconstruction techniques with noise and uncertain sensor locations," IEEE Transactions on Signal Processing, vol. 56, no. 8, pp. 3535-3547, Aug. 2008.

[7] G. H. Hardy, J. E. Littlewood, and G. Polya, Inequalities. London, UK: Cambridge University Press, 1959.

[8] H. A. David and H. N. Nagaraja, Order Statistics, 3rd ed. New York, NY: John Wiley \& Sons, 2003.

[9] R. Durrett, Probability: Theory and Examples, 2nd ed. Belmont, CA: Duxbury Press, 1996.

[10] A. W. van der Vaart, Asymptotic Statistics. Cambridge, UK: Cambridge University Press, 1998.

[11] Alan Oppenheim and Alan Willsky and Hamid Nawab, Signals and Systems, 2nd ed. USA: Prentice Hall, 1996. 